\title{
A Kriging constrained efficient global optimization approach applied to low-energy building design problems
}

\author{
E. Gengembre ${ }^{\mathrm{a} *}$, B. Ladevie ${ }^{\mathrm{b}}$, O. Fudym $^{\mathrm{b}}$ and A. Thuillier ${ }^{\mathrm{a}}$ \\ ${ }^{a}$ Company TBC Générateur d'Innovation, Colomiers 31770, France ${ }^{b}$ Université de Toulouse, \\ Mines Albi, CNRS, Centre RAPSODEE, Campus Jarlard, F-81013 Albi Cedex 09, France
}

\begin{abstract}
Low-energy building design is constrained not only by the total cost but also by both the energy demand and the comfort requirements. However, the evaluation of these criteria may require the implementation of time-consuming tasks, such as the direct building thermal simulation, which leads to difficulties in the design process. Moreover, it is of interest in this field to provide the designer with a large range of acceptable solutions rather than some unique optimal design. In this article, the application of an efficient global optimization approach is proposed as a tool to analyse the response functions of a building design problem. The method is based on a Kriging metamodel, which provides the global prediction of the objective and constraint functions, and an evaluation of uncertainty of the prediction at each point. The criterion for the infill sample selection is a generalized expected improvement function with desirable properties for stochastic responses. This criterion is maximized according to different constraints. First, inexpensive constraints are used as boundary constraints. Then, the expected violation criterion is used as a penalty. We use a particle swarm optimization algorithm to maximize the infill sample criterion according to the constraints. This approach is shown to be efficient for the building design problem, since the optimization is performed with an important reduction of the number of objective and constraint function calls. The Kriging metamodel is used to evaluate the sensitivity and the possible range of variations of the design parameters near their optimal values.
\end{abstract}

Keywords: building thermal design; building energy demand; Kriging; efficient global optimization

\section{Introduction}

In the building design process, the designer has to deal with different objectives and constraints and must intend to balance them by adjusting the design parameters of the building. The main problem arising is that each design parameter influences different indicators, which the designer must assess one by one.

The non-linear constrained optimization methods seem to be a convenient approach to solve these difficult multi-parameter and multi-indicator problems. Unfortunately, the direct simulation which computes the energy and comfort requirements may have a very

*Corresponding author. Email: egengembre@tbcinnovation.fr 
high cost in CPU time. Such problems are common in design optimization, where timeconsuming function evaluations are carried out by simulation tools. Classical optimization methods, based on derivatives, are not applicable because often derivative information is not available and is too expensive to approximate through finite differencing. Some powerful tools were developed like the optimization manager GenOpt [1], which implements different techniques such as the evolutionary algorithms, but requires thousands calls to the direct simulation model. Different approaches have been explored in order to solve this problem by reducing the computation time, for instance by building previously a regression function which will be used as a surrogate model by the optimization algorithm [2,3]. Such specific optimization methods have been developed, as the efficient global optimization (EGO) [4], which yields a global search and find an optimum while avoiding many calls to the expensive function. EGO makes use of the so-called Kriging metamodel [5] as a surrogate model [6,7]. The Kriging metamodel gives predictions at each point in the decision space, in order to decrease drastically the computational time of the expensive function.

The proposed approach aims to balance simultaneously the rate of improvement of the objective function, the exploration of the whole region of the domain which satisfies the constraints and the CPU time.

\section{Definition of the design problem}

At the beginning of the conception stage, it is assumed that some information is available for the designer, such as:

- the sketch, which is the list of volumes, surfaces and their orientation such as drawn by the architect,

- the geographical location, which is necessary to select the right weather file to be used in the simulation. The surrounding neighbourhood must also be known in order to determine the solar radiation and mask effects for each surface at every time step and

- the occupancy scenario, which is used to define the occupancy trends during a period of reference. The occupancy is coupled with a scenario of desired temperature in the building. The scenarios must be standardized in order to allow the comparison between different designs.

\subsection{Parameters}

To explore the use of optimization in conception, we will start with a very simple building and some general parameters for the early design stage.

Figure 1 is an illustration of the sketch in consideration herein, which is a single room building with a window on each vertical wall. The top of the south window is covered with a sunshade.

After manually translating this sketch in a database of orientated surfaces, we construct the direct model of the building depending on the following parameters:

- Window/wall ratio: rn, re, rs, rw, for north, east, south and west

Since the solar irradiation is different for each wall, it is necessary to use one ratio parameter for each wall. 


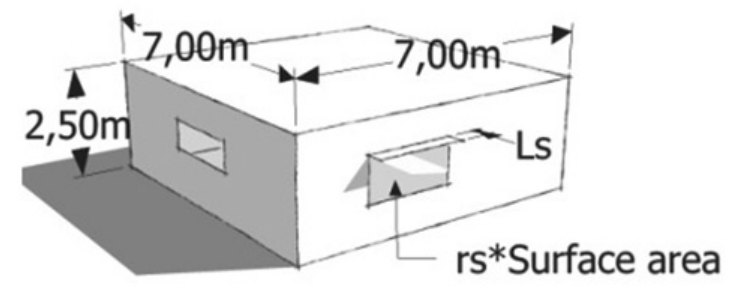

Figure 1. Sketch of the example.

- Global thermal resistance through opaque surfaces: $R$ in $\mathrm{m}^{2} \mathrm{~K} \mathrm{~W}^{-1}$

We will consider that the orientation of the opaque surfaces does not impact enough the heat transfer through the wall on average. However, it is possible to apply different insulation thickness on each surface if the global $R$ is satisfied.

- Thermal transmission through the windows: $\mathrm{Uw}$ in $\mathrm{W} \mathrm{m}{ }^{-2} \mathrm{~K}^{-1}$

The windows are assumed to have the same thermal transmission parameter Uw, but may have different dimensions. The solar transmission is not considered as a design parameter and is fixed to a previously chosen value in a constructive product database.

- Inertia: $\mathrm{Cm}$ in $\mathrm{kJ}^{-1} \mathrm{~K}^{-1} \mathrm{~m}^{-2}$ of floor

The inertia of the building can be described as a capacitor like in the $\mathrm{R} 5 \mathrm{C} 1$ [8] thermal model for global building simulation. This parameter must be improved in order to be used in a multi-zone model.

- Sunshade length above south window: Ls in $\mathrm{m}$

From this parameter, we can deduce the solar gain through the south window. To optimize a more complex sunshade, we require to use more parameters.

These parameters are used in the optimization to run the different indicators function. All other parameters are fixed in. We chose to limit the number of parameters in that study to focus on the optimization problem. Some other parameters as infiltration ratio will be added in further experiments.

\subsection{Indicators}

- Cost of the construction, $\mathrm{CC}\left(€ \mathrm{~m}^{-2}\right)$

This indicator is fundamental in helping the designer to design an efficient building without having to extend the budget. The cost is the sum of the different elements (walls, windows, etc.) and the systems implanted in the building.

- Energy consumption, EC (kWhpe $\mathrm{m}^{-2}$ per year)

The EC regroups the HVAC and lighting energy demands over one year. kWhpe is relative to $\mathrm{kWh}$ in primary energy. A yearly energy simulation of the building must be performed in order to evaluate these criteria, which make it time-consuming to evaluate.

- Cost of energy, CE (€ $\mathrm{m}^{-2}$ per year) 
As EC, this criterion is deduced from the results of a one year energy simulation.

- Life cycle cost assessment, LCC (€ $\mathrm{m}^{-2}$ per year)

This indicator is the sum of $\mathrm{CC}$ divided by the life time of the building and the yearly cost of energy, CE.

These are not the only design indicators used in building design. Thermal comfort or environmental impacts are more and more used to validate a building project. All the building indicators are not used in that study, but the method proposed has been thought in order to be compatible with all indicators that can be evaluated.

The designer must work within specific constraints. We can assume that the designer has a threshold for each indicator which will define the constrained decision space. In that decision space, the designer has to select a solution according to his wishes. Some preferences can be used as constraints: like to have a minimum windows ratio over all the building, or as a objective such as minimizing the life cycle cost assessment (LCC).

\subsection{Computation of $E C$ and $C E$ with the energy simulation model}

The energy simulation model allows the evaluation of the building's energy usage over the course of one year. We consider that the scenario of occupancy, the weather file and the sketch are fixed throughout the optimization process.

The building thermal model used in this study is the global normalized model ' $\mathrm{R} 5 \mathrm{Cl}$ ' which is used normatively in the French thermal regulation frame in order to assess the EC of the buildings [8].

The energy simulation model is used as a black box model which computes the heating and cooling demand, as well as the lighting consumption. The heating and cooling demand and lighting consumption are used to compute the EC and $\mathrm{CE}$ indicators, as shown in Figure 2. The approach is developed for future applications with dedicated professional

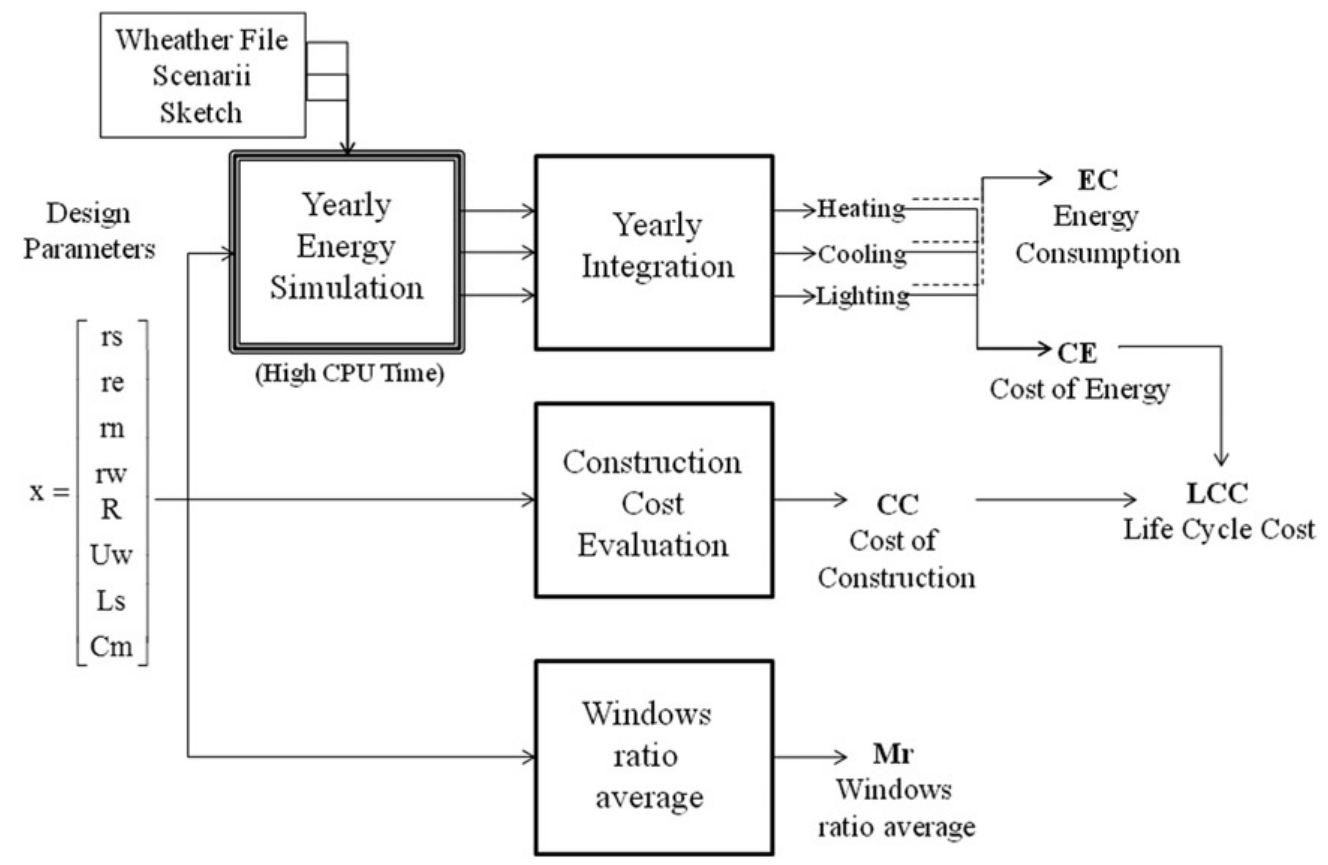

Figure 2. Flowchart of the evaluation of the indicators. 
simulation codes which computation time would be of various minutes or even hours. So it is necessary to reduce the call to the simulation in the optimization.

\subsection{Optimization using Kriging}

EGO is an optimization method which has been developed to find an optimum of an expensive function. We will consider energy simulation expensive to evaluate, because one simulation can be fast as a need of a few seconds, but thousands of simulations will take too much time for a efficient use in building conception.

The main idea of EGO is to replace the approximate expensive function by a predictor using a Kriging surrogate model for selecting the best next candidate to be evaluated by maximizing an infill sampling criterion (ISC) [9] which is detailed below.

Using that criterion allows to select the next best candidate in the space of the parameters according to the convergence to the goal and to improve the accuracy of the Kriging surrogate model.

As shown in Figure 3, optimization will update the Kriging surrogate model after each call to the expensive function in order to improve the accuracy of the surrogate model and to tend to the optimum.

To select the next sampling point, we maximize the ISC by another optimization which will be run for each iteration of EGO. The criterion is cheap to evaluate, so we use a global optimization like particle swarm optimization (PSO) to maximize it [10]. PSO has been already tested with other optimization techniques calling directly an energy building simulation program [11]. The performance trends of that study show that PSO give good results non-smooth simulation-based optimization with several local minima.

Different stopping criteria exist, but as in global optimization, it is impossible to be sure that the program reaches the optimum before all the space has been explored. In this study, the maximum number of simulation runs is used as stopping criterion.

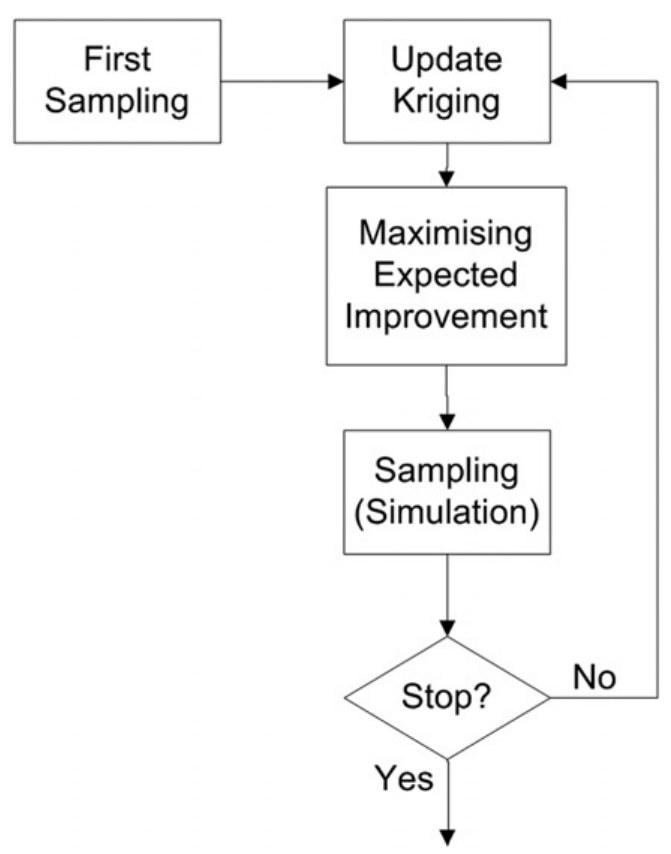

Figure 3. Flow chart of the EGO algorithm [4]. 
Obviously, this number will vary according to the number of parameters. In the application envisioned hereafter, the maximum number of simulation runs is 150 .

\subsubsection{Kriging metamodel}

The Kriging metamodel is a regression function which is corrected by a Gaussian process. The Kriging has been developed first for geostatistics [5]. With a classic regression, there is an error between the regression function and the true function. With the Kriging, the regression function $\hat{y}(x)$ and the true function $y(x)$ have the same value at each already evaluated point. Furthermore, if the correction is high for one evaluated point, there will be a good chance that the correction has to be high at the neighbourhood of that point and to estimate the local quality of the surrogate model in the space decision. That way the Kriging is a powerful tool to use in an optimization using surrogate models.

The predictor of a function $y(x)$ is built as in Equation (1).

$$
\hat{y}(x)=f(x)^{t} \beta+r(x)^{t} \gamma,
$$

where $x$ is a vector of the parameters. The first part of the equation represents a polynomial regression where $f(x)$ is a vector of the regression polynomial basis in $x$ and $\beta$ a vector of the regression polynomial coefficients. The second part represents the spatial correlation function with $r(x)$ the vector of the spatial correlation function and $\gamma$ the vector of the correlation coefficients.

With the construction of the surrogate model we can also estimate the variance of the predictor $\hat{\sigma}_{y}^{2}(x)$. It yields some information about the accuracy of the surrogate model at every point. We select a Gaussian correlation function depending on the spatial covariance parameters which can be fixed for each dimension of $x$, or optimized to maximize the accuracy of the Kriging.

If we try to minimize the surrogate model directly, the initial data sample should be really large to be accurate and to not converge to a local minimum. So, the EGO uses an ISC to select the new point to evaluate.

\subsubsection{Constrained ISC}

To select the next sampling point, we maximize the ISC by another optimization which will be run for each iteration of EGO. If the objective function depends on an expensive function, a Kriging surrogate model will be used to construct the expected improvement criterion $E_{I}^{y}(x)[12]$.

That criterion weights in balance the convergence of the minimization and the exploration of the function $y(x)$ as follows:

$$
\begin{aligned}
& E_{I}^{y}(x)=\left(Y_{\min }-\hat{y}\right) \cdot \Phi\left(\frac{Y_{\min }-\hat{y}}{\hat{\sigma}_{y}}\right)+\hat{\sigma}_{y} \cdot \varphi\left(\frac{Y_{\min }-\hat{y}}{\hat{\sigma}_{y}}\right), \quad \text { if } \hat{\sigma}_{y}>0, \\
& E_{I}^{y}(x)=0, \quad \text { if } \hat{\sigma}_{y}=0 .
\end{aligned}
$$

The functions $\hat{y}$ and $\hat{\sigma}_{y}$ depend on $x$ and are, respectively, the predictor of the function $y(x)$ and an estimation of the mean-squared error.

Several calls to $y(x)$ were necessary to build the predictor of $y(x)$ and $Y_{\min }$ is the minimum of these evaluations. 
$\varphi$ and $\Phi$ denote the probability density function and the cumulative distribution function for Gaussian mixture distribution, respectively. That criterion is high for space region where $\hat{\sigma}_{y}$ is high and $\hat{y}(x) \ll Y_{\min }$.

On the same model, an expensive constraint $g(x) \leq 0$ is translated as an expected violation, $E_{V}^{g}(x)[13]$, as follows:

$$
\begin{aligned}
& E_{V}^{g}(x)=(\hat{g}-0) \cdot \Phi\left(\frac{\hat{g}-0}{\hat{\sigma}_{g}}\right)+\hat{\sigma}_{g} \cdot \phi\left(\frac{\hat{g}-0}{\hat{\sigma}_{g}}\right), \quad \text { if } \hat{\sigma}_{g}>0, \\
& E_{V}^{g}(x)=0, \quad \text { if } \hat{\sigma}_{g}=0 .
\end{aligned}
$$

where $\hat{g}$ and $\hat{\sigma}_{g}$ depend on $x$ and are, respectively, the predictor of $g(x)$ and the estimation of the mean-squared error.

This criterion tends to zero when $\hat{g} \leq 0$ and is high when the constraint is violated or where there is a large uncertainty is the surrogate model.

After each Kriging construction, the expected improvement is maximized with a constraint on the expected violation using a PSO algorithm. A cheap constraint or objective depends on a cheap function, which is quickly evaluated. The cheap functions are directly used in the sub-optimization.

\section{Construction of the optimization problem}

For example, the method has been used on a specific problem of optimization:

$$
\text { Minimizing } y(x)=\operatorname{LCC}(x)=\frac{\mathrm{CC}(x)}{\text { lifespan }}+\mathrm{CE}(x) \text { with lifespan }=20 \text { years }
$$

According to

$$
\begin{aligned}
& g_{1}(x)=\mathrm{EC}(x) \leq 80 \mathrm{kWhpe}^{-2} \text { per year } \\
& g_{2}(x)=\mathrm{CC}(x) \leq 1500 \in \mathrm{m}^{-2} \\
& g_{3}(x)=\operatorname{Mr}(x) \geq 0.15 \text { with } \operatorname{Mr}(x)=\text { mean }(\mathrm{rn}, \mathrm{re}, \mathrm{rs}, \mathrm{rw})
\end{aligned}
$$

where $x$ is a vector of the parameters detailed previously. The selection of the objective function will orient the optimization problem in the constraint decision of space.

The constraints are much more important, because they define the constraints of the conception. It is possible that there is no solution, hence no decision space according to the constraints. If the constraint space of decision is large, the objective function will have an higher impact on the construction of the surrogate model in the space of solutions.

In that example, we use three kinds of constraint. The constraint on EC needs a call to the energy simulation. So that constraint will be used as the expected violation criterion presented earlier.

The constraint on CC and $\mathrm{Mr}$ are cheap to evaluate and will be used directly in the suboptimization. For the designer, $\mathrm{CC}$ is a constraint of this project and $\mathrm{Mr}$ is a preference on the conception.

\subsection{Used constrained ISC}

That criterion is maximized at each iteration using the PSO optimization using thousand iterations with 50 particles. The constraints are used as penalty in order to orient the 
Table 1. Parameter range.

\begin{tabular}{lccc}
\hline$x$ & Minimum & Maximum & Grid step \\
\hline rs & 0 & 0.9 & $10^{-2}$ \\
re & 0 & 0.9 & $10^{-2}$ \\
rn & 0 & 0.9 & $10^{-2}$ \\
rw & 0 & 0.9 & $10^{-2}$ \\
$R$ & 2 & 6 & $10^{-2}$ \\
Uw & 1.00 & 1.80 & $10^{-2}$ \\
Ls & 0 & 0.50 & $10^{-2}$ \\
Cm & 50 & 370 & 10 \\
\hline
\end{tabular}

particles of the PSO optimization algorithm to the constrained decision space. The used infill sampling criterion, $\operatorname{ISC}(x)$, is written as follows:

$$
\mathrm{ISC}=E_{I}^{\mathrm{LCC}}-C s t r_{\mathrm{E}}^{\mathrm{EC}}-C s t r^{\mathrm{CC}}-C s t r^{\mathrm{Mr}} .
$$

where ISC and all its components depend on $x . \operatorname{Cstr}_{E}(\cdot)$ means estimated constraint, which is used to estimate the violation of the EC constraint and $C \operatorname{str}(\cdot)$ means constraint, which is used as a penalization to avoid exploration outside the space of solutions.

$$
\begin{gathered}
\left.C s t r_{E}^{\mathrm{EC}}=\left[\left(E_{V}^{\mathrm{EC}}>0.01\right)\right)+E_{v}^{\mathrm{EC}}\right] \cdot 10^{5} . \\
C s t r^{\mathrm{CC}}=[(C C>1500)+\min (0, C C-1500)] \cdot 10^{10}, \\
\operatorname{Cstr}^{\mathrm{Mr}}=[(C C<0.15)+\min (0,0.15-\mathrm{Mr})] \cdot 10^{10},
\end{gathered}
$$

with $\mathrm{Mr}=$ mean (rs, re, rn, rw)

$E_{\mathrm{I}}^{\mathrm{LCC}}(x)$ depends on $\mathrm{CC}$ which is directly computed and $\mathrm{CE}$ which is predicted via the Kriging. So $E_{I}^{\mathrm{LCC}}(x)$ is used using only $\hat{\sigma}_{\mathrm{CE}}$, the estimated standard deviation of the error on CE.

\subsection{Performing the optimization algorithm}

The optimization is initiated with 12 thermal simulations which are determined by a design of experiment table L12 from Taguchi [14] even if the point is out of the constrained space of decision according to the cheap constraints. That choice has been made according to the fact that a surrogate model will be more accurate in interpolation rather than extrapolation [15].

The parameters are real numbers and continuous. According to the required accuracy, the allowed values for each parameter are chosen on a grid. Their range and grid step are fixed as shown in Table 1. They are limited by the technology of the different components of the building. We detail the 12 points simulated for the initialization in Table 2.

\section{Discussion and result analysis}

The optimization has been realized with 150 calls to the simulation. The best solution was number 87 . Another try was used with 1000 calls to the simulation with no better results. 
Table 2. Experiment table L12 applied on the parameters.

\begin{tabular}{lrrrrrrrrrrrr}
\hline$x$ & 1 & 2 & 3 & 4 & 5 & 6 & 7 & 8 & 9 & 10 & 11 & 12 \\
\hline rs & -1 & -1 & -1 & -1 & -1 & -1 & 1 & 1 & 1 & 1 & 1 & 1 \\
re & -1 & -1 & -1 & 1 & 1 & 1 & -1 & -1 & -1 & 1 & 1 & 1 \\
rn & -1 & -1 & 1 & -1 & 1 & 1 & 1 & 1 & -1 & 1 & -1 & -1 \\
rw & -1 & -1 & 1 & 1 & -1 & 1 & 1 & -1 & 1 & -1 & 1 & -1 \\
$R$ & -1 & -1 & 1 & 1 & 1 & -1 & -1 & 1 & 1 & -1 & -1 & 1 \\
$\mathrm{Uw}$ & -1 & -1 & -1 & -1 & 1 & 1 & -1 & 1 & 1 & -1 & 1 & -1 \\
$\mathrm{Ls}$ & -1 & 1 & -1 & 1 & -1 & 1 & 1 & 1 & -1 & -1 & -1 & 1 \\
$\mathrm{Cm}$ & -1 & 1 & -1 & 1 & 1 & -1 & 1 & -1 & 1 & 1 & -1 & -1 \\
\hline
\end{tabular}

Table 3. Best evaluation.

\begin{tabular}{lc}
\hline Indicators & Results \\
\hline LCC $\left(€ \mathrm{~m}^{-2}\right.$ per year $)$ & 84.5 \\
EC $\left(\mathrm{kWhpe}^{-2}\right.$ per year $)$ & 61.3 \\
$\mathrm{CC}\left(€ \mathrm{~m}^{-2}\right)$ & 890 \\
$\mathrm{Mr}$ & 0.15 \\
\hline
\end{tabular}

Table 4. Computed optimal parameters.

\begin{tabular}{lccc}
\hline$x$ & $\begin{array}{c}\text { Predicted } \\
\text { minimum value }\end{array}$ & $\begin{array}{c}\text { Computed optimal } \\
\text { parameters }\end{array}$ & $\begin{array}{c}\text { Predicted } \\
\text { maximum value }\end{array}$ \\
\hline $\mathrm{rs}$ & $0.39 \mathrm{Mr}$ & 0.39 & $0.51 \mathrm{EC}$ \\
$\mathrm{re}$ & 0 & 0 & $0.24 \mathrm{EC}$ \\
$\mathrm{rn}$ & $0.21 \mathrm{Mr}$ & 0.21 & $0.59 \mathrm{EC}$ \\
$\mathrm{rw}$ & 0 & 0 & $0.25 \mathrm{EC}$ \\
$R$ & $2.16 \mathrm{EC}$ & 3.16 & $5.89 \mathrm{CC}$ \\
$\mathrm{Uw}$ & 1.00 & 1.24 & 1.80 \\
$\mathrm{Ls}$ & 0 & 0.35 & 0.50 \\
$\mathrm{Cm}$ & $221 \mathrm{EC}$ & 370 & 370 \\
\hline
\end{tabular}

It is difficult to determine when the optimization has reached the global optimum, so we will just talk about the best evaluation. That kind of optimization has already been tested on several functions $[9,13,15]$. Rather than comparing the performance of EGO to other optimization algorithm, we will propose a new way to use the surrogate model in a design context, specifically with constraints.

The first result of the optimization is the best solution which has been evaluated as shown in Table 3. The only active constraint is on $\mathrm{Mr}$, because $\mathrm{Mr}$ is equal to the threshold.

Another result is the application of surrogate model. By using the surrogate model, it is possible to estimate the space of the solution to quickly give some information to the designer. In Table 4, the parameters of the optimum are given, and the freedom around each parameter. So if the designer wants to modify the best solution, he will have to respect 
the minimum-maximum value to respect the constraints. $\mathrm{EC}, \mathrm{CC}$ and $\mathrm{Mr}$ are, respectively, the energy consumption, the construction cost and the mean window ratio constraint indicator. The minimum value of $\mathrm{rs}$ is limited by the constraint on $\mathrm{Mr}$ and the maximum by the constraint on EC.

To describe the space of the solution to the designer, the idea is that the designer will only modify one parameter at a time. So the parameters range depends on the starting point. For each minimum or maximum, it is possible to know which constraint is reducing the space of the solutions. We can see in Table 4 that EC constraint is most restrictive. To quickly evaluate the EC constraint, we used the EC surrogate model.

For each minimum or maximum estimated, a simple constrained optimization has been used. By using the surrogate model, the estimation needs less than a second on a regular desktop. Without it, the same estimation would have needed a large number of calls to the simulation for each optimization.

To illustrate how a designer wants to explore around a solution, we will increase re from 0 to 0.24 , which means installing another window on the east wall (Table 5). We are not at the best solution anymore but the range of the parameters according to the constraint can be evaluated again.

By forcing a parameter close to the constraint, the space of the solution around the new solution is much narrower. But just using the surrogate model can be dangerous, because the accuracy of the surrogate model is dependent on the position in the space of the solution.

In Table 6, we compare the predicted value of the parameters and the true value by using the simulation. Only EC and LCC depend on predictors. The true value and the predicted value are close, but $\mathrm{EC}_{\text {predicted }}$ is according to the constraint and $\mathrm{EC}_{\text {true }}$ is not. That problem can be explained by the fact that region has not been explored enough

Table 5. Modified parameters.

\begin{tabular}{lccc}
\hline$x$ & $\begin{array}{c}\text { Predicted } \\
\text { minimum value }\end{array}$ & $\begin{array}{c}\text { Parameter } \\
\text { value }\end{array}$ & $\begin{array}{c}\text { Predicted } \\
\text { maximum value }\end{array}$ \\
\hline rs & $0.15 \mathrm{Mr}$ & 0.39 & $0.39 \mathrm{EC}$ \\
re & 0 & 0.24 & $0.24 \mathrm{EC}$ \\
rn & 0 & 0.21 & $0.22 \mathrm{EC}$ \\
rw & 0 & 0 & 0.00 \\
$R$ & $3.07 \mathrm{EC}$ & 3.16 & $4.99 \mathrm{EC}$ \\
$\mathrm{Uw}$ & 1.00 & 1.24 & $1.46 \mathrm{EC}$ \\
$\mathrm{Ls}$ & $0.22 \mathrm{EC}$ & 0.35 & 0.50 \\
$\mathrm{Cm}$ & $364 \mathrm{EC}$ & 370 & 370 \\
\hline
\end{tabular}

Table 6. Modified parameters indicators.

\begin{tabular}{lcc}
\hline Indicators & Predicted value & True value \\
\hline LCC $\left(€ \mathrm{~m}^{-2}\right.$ per year $)$ & $91.0(3 \%)$ & 93.3 \\
EC $\left(\mathrm{kWhpe}^{-2}\right.$ per year $)$ & $77.0(4 \%)$ & 80.8 \\
CC $\left(€ \mathrm{~m}^{-2}\right)$ & 892 & 892 \\
$\mathrm{Mr}$ & 0.21 & 0.21 \\
\hline
\end{tabular}


through the optimization. The error is about $4 \%$ for the EC constraint. So the accuracy is enough to give an orientation the designer, but not enough to avoid to call the simulation especially near constraints.

The best solution would be to use the surrogate model to estimate the space of solution and to call the simulation for each new point given by the designer, and then update the surrogate model.

To represent how the surrogate model fits the true function, Figures 4 and 5 illustrate the surrogate model on one parameter. The other parameters are fixed to the best solution obtained.

In Figure 4, the best iteration is really close to the minimum of LCC depending on $R$. The two constraints are not active on $R$. The accuracy of the surrogate model is low where the exploration was avoided, because the constraint prevents the exploration on that region or because the expected improvement is smaller.

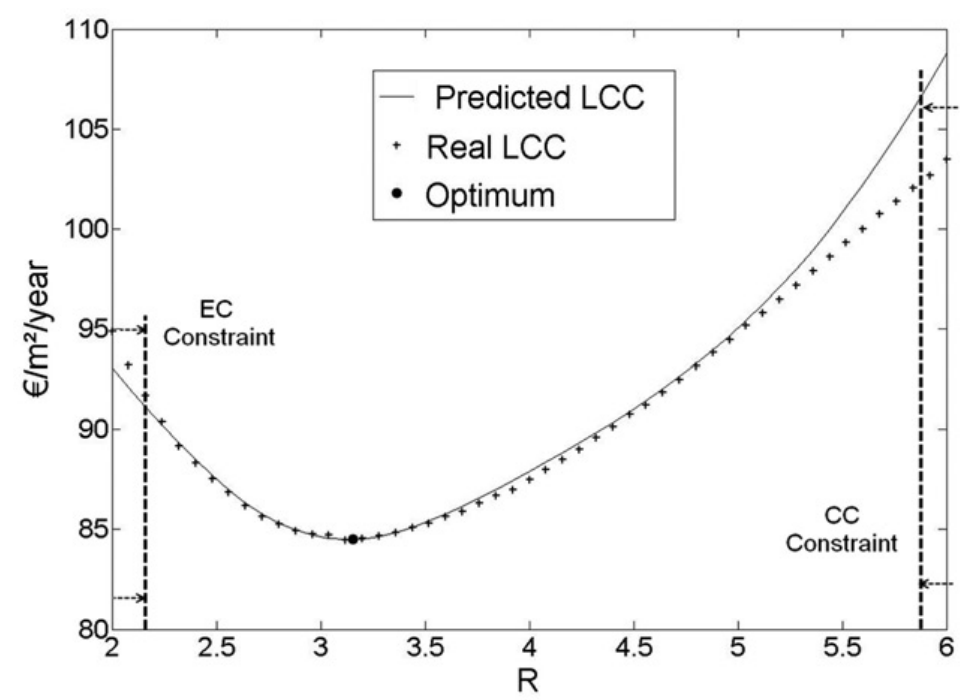

Figure 4. LCC and predicted LCC depending on $R$.

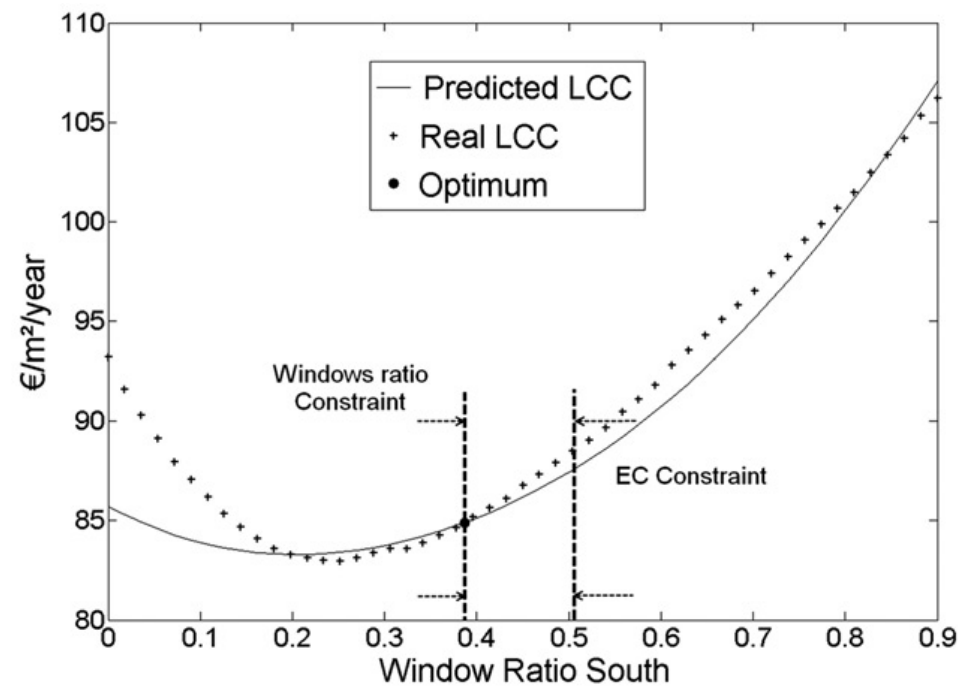

Figure 5. LCC and predicted LCC depending on rs. 
In Figure 5, the constraint $\mathrm{Mr}$ is obviously preventing the exploration close to the real optimum on that parameter. The surrogate model accuracy is not so good, but gives enough information to orient the designer on the space of solution.

To realize that comparison, we needed to realize 50 simulations by parameter, with the surrogate model the 50 evaluations were almost immediate.

To orient the designer, it is possible to give much more detailed information. With a surrogate model on each output of the simulation, it will be possible to estimate the impact of each parameter on the heating demand, the cooling demand and the lighting consumption quickly.

Another way which has not been shown here is to always look for an optimum when the designer is modifying the constraint or fixing a parameter.

\section{Conclusion}

EGO applied to a building design problem is shown to be suitable to save a large computational time by reducing the number of calls to the expensive (objective and constraints) functions computation in comparison with other widespread usual methods. This is achieved by building and using a Kriging metamodel which is used instead of the full energy building simulation for the optimization approach. The Kriging metamodel also yields the range of authorized values for the design parameters within the constraint limits and is user-friendly to predict the effect of changing the value of the design parameters without the necessity of running more direct simulations.

With the estimation of the parameters range according to the constraints limits, the designer can estimate which design of the building is possible.

The proposed approach has been implemented and analysed in a basic standard monozone case with a global energy simulation model. Next steps of this study will be to implement a multi-objective approach and to define a how to link the global design parameters of a more complex case to the local constructive parameters defined in a database.

\section{Nomenclature}

Criteria used in the optimization problem

$$
\begin{aligned}
\text { EC energy consumption }\left(\mathrm{kWhpe}^{-2} \text { per year }\right) \\
\mathrm{CE} \text { cost of the energy }\left(€ \mathrm{~m}^{-2} \text { per year }\right) \\
\text { LCC life cycle cost assessment }\left(€ \mathrm{~m}^{-2} \text { per year }\right) \\
\mathrm{Mr} \text { mean of the window ratios }
\end{aligned}
$$

Kriging surrogate model

$X \quad$ vector of the parameters

$f(x) \quad$ vector of the regression polynomial basis

$\beta \quad$ vector of the regression polynomial coefficients

$r(x)$ vector of the spatial correlation function

$\gamma \quad$ vector of the correlation coefficients

$\hat{\sigma}^{2}(x) \quad$ estimation of the variance of the predictor 
Efficient global optimization

$y(x)$ true expensive objective depending on $x$

$g(x)$ true expensive constraint depending on $x$

$Y_{\min }$ minimum value evaluated with $y(x)$

$E_{I}^{y}(x) \quad$ expected improvement on $y(x)$

$E_{V}^{g}(x)$ expected violation on $g(x)$

$\operatorname{ISC}(x)$ infill sampling criterion

Parameters of the thermal simulation

rn, re, rs, rw window/wall ratio: for north, east, south and west

$R$ global thermal resistance through opaque surfaces in $\mathrm{m}^{2} \mathrm{~K} \mathrm{~W}^{-1}$

$U w$ thermal transmission through the windows in $\mathrm{W} \mathrm{m}^{-2} \mathrm{~K}^{-1}$

$C m$ inertia in $\mathrm{kJ} \mathrm{K}^{-1} \mathrm{~m}^{-2}$ of floor

Ls sunshade length above south window in $\mathrm{m}$

\section{Acknowledgements}

The authors acknowledge the financial support of the French National Research Agency (ANR), as part of the AMMIS project.

\section{References}

[1] M. Wetter, GenOpt a generic optimization program, Proceedings of the 7th International Building Simulation Conference, Rio de Janeiro, Brazil, 2001.

[2] I. Jaffal, C. Inard, and C. Ghiaus, Fast method to predict building heating demand based on the design of experiments, Energy Build. 41 (2009), pp. 669-677.

[3] F. Pernodet, H. Lahmidi, and P. Miche, Use of Genetic Algorithms for Multicriteria Optimization of Building Refurbishment, Proceedings of the 11th International Building Simulation Conference, Glasgow, Scotland, 2009.

[4] D.R. Jones, M. Schonlau, and W.J. Welch, Efficient global optimization of expensive black-box functions, J. Global Optim. 13 (1998), pp. 455-492.

[5] G. Matheron, Principles of geostatistics, Econ. Geol. 58 (1963), pp. 1246-1266.

[6] D.R. Jones, A taxonomy of global optimization methods based on response surfaces, J. Global Optim. 21 (2001), pp. 345-383.

[7] A.I.J. Forrester and A.J. Keane, Recent advances in surrogate-based optimization, Progr. Aerosp. Sci. 45 (2009), pp. 50-79.

[8] Réglementation Thermique 2005: Méthode de calcul Th-CE, CSTB, 2006. Available at www.rtbatiment.fr/fileadmin/documents/RT2005/pdf/Methode_Th_CE.pdf, last access 20/02/12.

[9] J.M. Parr, C.M.E. Holden, A.I.J. Forrester, and A.J. Keanea, Review of Efficient Surrogate Infill Sampling Criteria with Constraint Handling, Second International Conference on Engineering Optimization, Lisbon, Portugal, 2010.

[10] B. Birge, PSOt, A Particle Swarm Optimization Toolbox for Matlab, IEEE Swarm Intelligence Symposium Proceedings, Indianapolis, IN, 2003.

[11] M. Wetter and J. Wright, A comparison of deterministic and probabilistic optimization algorithms for nonsmooth simulation-based optimization, Build. Environ. 39 (2004), pp. 989-999.

[12] J. Mockus, Application of Bayesian approach to numerical methods of global and stochastic optimization, J. Global Optim. 4 (1994), pp. 347-365. 
[13] C. Audet, J. Dennis, D. Moore, A. Booker, and P. Frank, Surrogate-model-based Method for Constrained Optimization - LACSI at Rice University, AIAA/USAF/NASA/ISSMO Symposium on Multidisciplinary Analysis and Optimization, Long Beach, CA, 2000.

[14] M. Pillet, Les Plans d'expériences par la Méthode Taguchi, Les Editions d'organisation, Paris, 1997.

[15] M.J. Sasena, Flexibility and efficiency enhancements for constrained global design optimization with Kriging approximations, Ph.D. diss., University of Michigan, MI, 2002. 